\title{
Simple waveguide model of arbitrary filled plane-plane cavity
}

\author{
S. Anokhov \\ International Center "Institute of Applied Optics" of NAS of Ukraine \\ 10-G Kudryavskaya str., 254053, Kyiv, Ukraine \\ phone: (38044) 212-21-58; fax: (38044) 212-48-12 \\ e-mail:khizh@lomp.ip.kiev.ua
}

\begin{abstract}
Some waveguide_features responsible for angular structure of a plane-plane cavity output emission, which were not discussed before, are considered. A number of common regularities in mode structure of such cavity at the level of their elementary organization is established. Among them are: the angular quantization of initial plane waves spectrum, the real size of diffraction losses, the phenomenon of reorientation of plane waves in waveguide propagation, the role of optical filling of the cavity, etc. The obtained data most essentially supplement the traditional representations of mode properties in the area of the small cavity apertures characterized by the Fresnel number about or less than unity.
\end{abstract}

Keywords: cavity, waveguide, mode, optical length, effective length, losses.

Paper received 27.04.00; revised manuscript received 15.05.00; accepted for publication 16.06.00.

\section{Introduction}

Though all general laws of open cavity operation are considered as have been established [1], the properties of real modes of laser emission still remain a subject of active researches. In many respects it is explained by the break existing between the theoretical model of open cavity, which is generally considered as optically homogeneous, and the specificity of any laser system with a particular combination of optical elements. Naturally, such model simplification may cause the loss of important aspects of mode real formation process. It, in particular, also explains an often discrepancy between experimental data with theoretical predictions.

The detection of structural anomalies in the far field of the single-mode $\mathrm{Nd}^{+}$: glass laser with plane mirrors and intracavity slit $[2,3]$ gave the cause for the present article. Though these anomalies had obviously regular character, which had not been connected with the action of random factors, any explanation within the limits of existing theoretical conceptions $[1,4,5]$ was not found. Therefore, the immediate purpose of the article was an attempt to construct a simple physical model of arbitrary filled plane-plane cavity, which could give an opportunity to analyze quantitatively the angular structure of modes that it forms.

\section{Waveguide approach to description of an open cavity}

The Wainshtein's idea about the analogy between an open cavity and a segment of a waveguide, mathematical model of which was well developed [1,5], became very fruitful for the theory of optical cavities. The field of any mode in such cavity can be represented by the superposition of plane waves converging under a small angle on its axis. As is known from optics, the similar superposition has steady spatial structure, where number of field extrema on the aperture of the cavity is determined by the particular convergence angle of plane waves. For the further consideration let us use the «semi-geometrical» approach proposed in [1], which combines beam representation of waves inside the cavity with their diffraction transforms at the open edge.

The general scheme of plane waves propagation in this case is shown in Fig. 1a. The two-dimensional cavity represented here contains simultaneously 4 plane waves equally inclined relatively to the optical axis. The addition by pairs of 1-2 and 3-4 waves gives two waveguide waves propagating in the cavity upwards and downwards in parallel to the mirrors (Fig. 1b). The superposition of close in direction waves $2-3$ and 1-4 forms light beams propagating along the optical axis (Fig. 1c). As the real 


\section{S. Anokhov: Simple waveguide model of arbitrary filled plane-plane cavity}

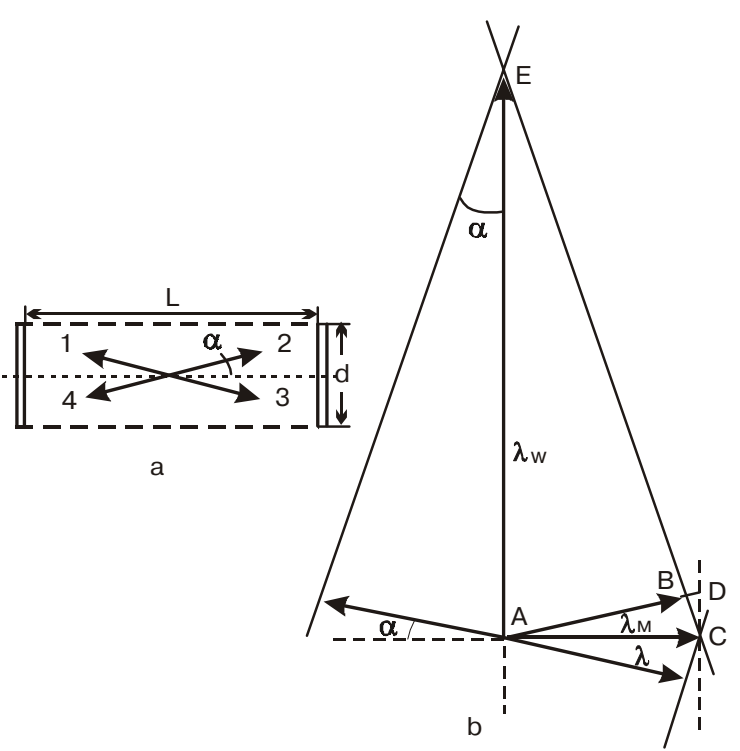

Fig. 1. Schematic representation of plane waves in the cavity (a) and their aggregation into a light mode $\left(\lambda_{\mathrm{M}}\right)$ and into a guide wave $\lambda_{\mathrm{w}}(\mathrm{b})$, where $\mathrm{AB}$ and $\mathrm{EC}$ is the normal and the front of plane wave.

convergence angles of plane waves in the cavity are of small value: $\alpha \sim 10^{-3} \div 10^{-5}$, the distinction in lengths between light and guide waves is from 3 up to 5 orders of magnitude.

The least obvious moment here is the reflection of plane waves from the open edge of the cavity. The reflection effect for the incident wave, which interacts with the edge, is caused by its diffraction on the aperture edges of the cavity, and is similar to the diffraction of light on a periodic pattern, just with the difference that the slit in this case is only one (the open edge under the grazing incidence angle), but the waves are numerous (the train, repeatedly «combined» in the cavity). The separation of the total field maximum in the direction of the plane wave mirror reflection from the aperture boundary of the cavity becomes the result of this process [1].

A necessary condition of the effect is the severe periodicity of any wave front interaction moments for each aperture edge. For this any normal of the initial wave should propagate in the cavity by one of closed trajectories, which are self-reproducing relatively the transversal pass around the cavity (Fig.2a-c). All such trajectories satisfy the condition

$d=k \alpha L_{e}$,

where $k=1,2,3 \ldots$ - the number of double passes between mirrors during the wave round path in the cavity, $\alpha$ - the angle of wave incidence onto the mirror (the inclination angle of the wave), $L_{e}=\Sigma l_{i} / n_{i}$ - the effective length of the cavity, where $l_{i}$ - its homogeneous parts with constant value of refraction index $n_{i}[6]$.

As is seen, the trajectory an arbitrary wave normal forms the set of similar ring elements (Fig. 2), the number
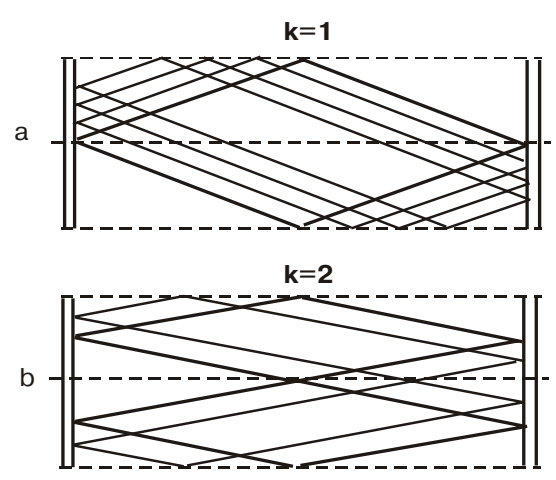

$\mathbf{k}=\mathbf{3}$

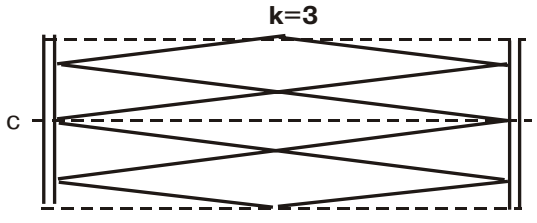

Fig. 2. Normal trajectories of plane waves at small values of $k$ parameter (for details see the text).

of which coincides with value of $k$ index. The last should not be confused with the transversal index of modes - at the inclinations of waves which does not exceed the diffractive divergence of the emission, all variants of the trajectories correspond to the fundamental modes of the cavity.

At implementation of the condition (1) and for small angles of wave deviation from the axial direction of propagation, the significant part of their energy is reflected by the open edges and remains in the cavity. The reflectance of the guide wave is represented by the simple formula [5]:

$A=-\exp \left[-\alpha \beta(1-i)\left(2 \pi L_{\mathrm{o}} / \lambda\right)^{1 / 2}\right]$,

where $A$ - the relative amplitude of the reflected wave, $\beta=0.824, L_{\mathrm{o}}=\Sigma l_{i} n_{i}-$ the optical length of the cavity, $\lambda$ - the length of a plane wave in vacuum. The phase multiplier in the right part of the formula means the phase shift of the guide wave at reflection, which is possible to be interpreted as a small displacement of the reflecting plane beyond the aperture boundary of the cavity $[1,5]$.

In view of stated, an arbitrary open cavity at the twodimensional representation can be described as a rectangular cavity with two pairs of both real and virtual reflecting boundaries. Each of plane waves, being transformed during the reflection from one into another, seems to be closed in the resonant cavity. A simple and evident interpretation of this situation can be given. As is known, the interference of unlimited plane waves forms two mutually perpendicular systems of nodal planes with zero field intensity. Combining the boundaries of the resonant cavity with the appropriate planes, we receive the limited space with the same structure of the confined light field. The two-dimensional images in Fig. 2 correspond to an arbitrary section of such a cavity. 


\section{S. Anokhov: Simple waveguide model of arbitrary filled plane-plane cavity}

We shall consider for the definiteness the cavity with a slit aperture, located at one of mirrors. This cavity is similar to that used in the experiments mentioned in the introduction $[2,3]$. Its optical scheme is completely equivalent to the cavity with one strip mirror and unlimited another one (Fig. 3). The structure of transversal oscillations in it corresponds to the symmetric strip cavity of double length [5], where during the complete pass of the cavity by a plane wave, the mode makes $2 k$ passes with the period of $2 L_{\mathrm{o}} / c$. Subject to this the complete number of double passes of plane waves in the cavity and their edge power reflectance can be presented, accordingly, by formulae

$k=d / 2 \alpha L_{e}$,

$R_{d}=\exp \left[-4 \alpha \beta\left(\pi L_{\mathrm{o}} / \lambda\right)^{1 / 2}\right]$.

After the substitution in (3)-(4) of parameter values which are typical for $\mathrm{Nd}+$ : glass lasers: $d=2 \mathrm{~mm}, \alpha=$ $2 \cdot 10^{-4}, L_{\mathrm{o}}=500 \mathrm{~mm}, L_{e}=400 \mathrm{~mm}, \lambda=1060 \mathrm{~nm}$, we receive $k=12, R_{d}=0.58$.

As it was already noted, the length of the guide wave coincides with the period of fringes at the interference of plane waves. It is essential, that the structure of these fringes does not depend on the presence of optical elements in the cavity: at passage of a superposition of plane waves through a boundary of two media the width of fringes $\lambda / 2 \alpha$ is kept due to the proportional change of $\lambda$ and $\alpha$ values in each medium. It follows from this that at any optical filling of the cavity the guide wave fronts do not suffer distortion - the cavity remains homogeneous for them. This fact completely equalizes the waveguide representation of both empty and arbitrary filled cavities and makes it possible to spread the well-studied laws of waveguide oscillations in the ideal cavity $[1,5]$ onto the last one.

\section{Phase matching of waves}

There is a value in the theory of cavities, which has no analogs in usual optics that sometimes complicates its clear

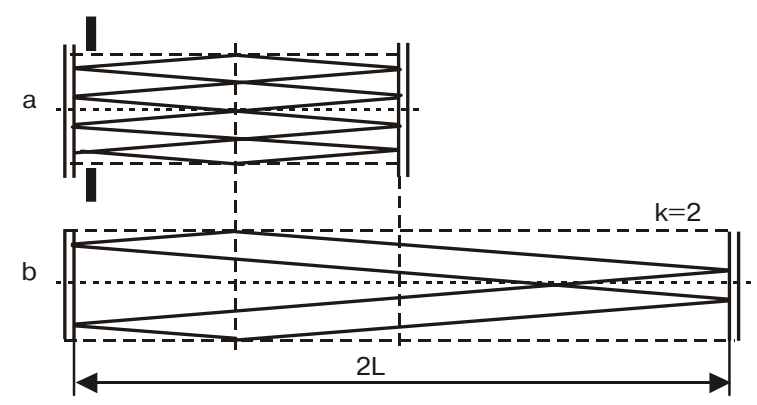

Fig. 3. Scheme of the cavity with one-side aperture diaphragming. perception. It concerns to the phase correction for modes revealed at the rigorous analysis of field intracavity oscillations [1,5]. The waveguide approach allows to consider features of this phenomenon in the most evident way.

As it follows from the simple geometrical consideration, the phase incursion for the plane waves and for the modes they form in the cavity is different [1]. Really, the wavelength of the total field of waves $\lambda_{\mathrm{M}}$ exceeds the length of each of them in $1 / \cos \alpha \approx\left(1+\alpha^{2} / 2\right)$ times, that is illustrated by the ratio of $\mathrm{AD} / \mathrm{AB}$ segment in Fig. 1b. The mode spatial retardation associated with it equals to $2 \pi(1-$ $A B / A D) \approx 2 \pi \alpha^{2}$ phase shift for one elementary wave field oscillation. For double pass of waves between the mirrors this retardation becomes equal to $2 \pi \alpha^{2} 2 L_{\mathrm{o}} / 1\left(1+\alpha^{2} / 2\right) \approx$ $4 \pi L_{\mathrm{o}} \alpha^{2} / \lambda$, and for the complete round pass of the cavity it increases $2 k$ times, reaching

$\Delta=4 \pi L_{\mathrm{o}} \alpha d / \lambda L_{e}$

The phase correction for modes, usually used in the theory, is required for calculation of exact values of their frequencies [1,5], here coincides with the value of a mode phase increment retardation for the pass of the cavity relatively to the increment of a plane wave following the same direction:

$\delta=2 \pi L_{\mathrm{o}} \alpha^{2} / \lambda$

The meaning of the found $\Delta$ value is another. The energy state of the field in the cavity depends on complete phase shift of the mode relatively to its plane components. The most favorable situation in this respect is the determined by the equation

$\Delta_{m}=2 \pi(m+1)$,

where $m=0,1,2 \ldots$ If the condition ( 7 ) is obeyed, the phase self-coordination simultaneously for all light fields is reached in the cavity. In this case the angle between the plane wave normals and the axis according to (6) is represented by the formula

$\alpha_{m}=\lambda L_{e}(m+1) / 2 L_{\mathrm{o}} d$.

Beside the negligible multiplier the received value coincides with plane waves convergence angle of the empty cavity mode, where $m$ - transversal index of the mode $[1,5]$. At usual for solid-state lasers values of parameters $L_{\mathrm{o}}=500 \mathrm{~mm}, L_{e}=400 \mathrm{~mm}, \lambda=1060 \mathrm{~nm}, d=2.0 \mathrm{~mm}-$ the formula (9) for $m=0$ gives $\alpha_{\mathrm{o}}=2 \cdot 10^{-4}$.

From the formulae (3) and (9) it is not difficult to find the connection between indexes $k$ and $m$ :

$k_{m}=d^{2} L_{\mathrm{o}} / \lambda L_{e}^{2}(m+1)$

Parameter $k_{m}$ determines here the number of wave double passes in the cavity at the mirrors incidence angle of $\alpha_{m}$. The number of passes grows in process of $m$ value reduction, reaching the maximum at $m=0$, when the inclination of plane waves according to (8) is minimal. 


\section{S. Anokhov: Simple waveguide model of arbitrary filled plane-plane cavity}

\section{Diffraction losses}

The waveguide approach gives the most simple interpretation of plane-plane cavity diffraction losses, connecting them with the light power which is lost at diffractive reflection of guide waves from the open edge $[1,5]$. To have an opportunity to compare quantitatively these losses with other ones (radiation output through mirrors, scattering on optical heterogeneity, absorption, etc.) it is necessary to use their values appropriate to plane wave double pass in the cavity [1].

For this purpose we shall pass from units of power $R_{d}$ to exponential parameter of losses $\gamma=-\ln R_{d}$. As it was already specified, the closed wave trajectory contains $2 k$ of plane wave double passes between mirrors and two diffractive reflections from its aperture boundaries. In view of this the parameter of a wave diffractive losses, which corresponds with its two passes between mirrors, is determined by the ratio $\gamma_{d}=-2 \ln R_{d} / 2 k$ or

$\gamma_{d}=8 b a^{2} L_{e}\left(p L_{\mathrm{o}} / 1\right)^{1 / 2} / d$

At the typical conditions of the cavity's operation the resonant condition $\alpha=\alpha_{m}$ is obviously met, therefore it is possible to write the formula (10) as

$\gamma_{d}=\beta(\pi / 2)^{2}(m+1)^{2}\left(L_{e} / L_{\mathrm{o}}\right)^{3 / 2} / 8 N^{3 / 2}$,

where

$N=d^{2} / 8 \lambda L_{e}$

- is the Fresnel parameter of the arbitrary filled cavity with one-sided aperture. As is seen, in contrast to the Fresnel number for the similar empty cavity, the parameter (12) contains the multiplier $\left(L_{e} / L_{\mathrm{o}}\right)^{3 / 2}$. At the growth of the cavity optical filling, which means the increase of distinction between lengths $L_{e}$ and $L_{\mathrm{o}}$ in favor of the last, the diffractive losses reduce.

The formula (11) makes clear the reason of failures with the practical realization of «large effective length» cavities - one of the most enticing offers for angular selection of laser radiation. The idea consists in introduction of a telescopic system into a plane cavity to reduce its angular aperture up to just one Fresnel zone without the vignetting of an active element [1]. Thus, the considerable growth of the cavity effective length $L_{e}$ is reached at $L_{\mathrm{O}}=$ const, which, as is clear now, results in keen $\left(\sim L^{3 / 2}\right)$ growth of diffractive losses. These losses become the stumbling block for any attempts to change excessively the natural balance between values of $L_{e}$ and $L_{\mathrm{o}}$.

\section{Effect of waveguide trajectory reorientation}

One of the key parameters of waveguide mode representation is $k$ - the number of wave double passes in the cavity. On its sense $k$ has an integer value in principle. Let us consider what will happen with the light field structure in the cavity at smooth change of its aperture or length? The answer is unequivocal: the angle between plane wave normals and the axis will evolve in this case an oscillatory manner, where its monotonous change according to the formula (8) should be periodically interrupt by fast returning into the area of optimal values with the change of $k$ value on one unity.

The spasmodic change of plane wave inclination means the system transition from one stationary condition into another with the change wave pass number in the cavity. Thus, the configuration of the wave trajectory (Fig. 2b-c) changes by jump on one ring element with complete reorientation wave normals in the cavity. It is logical to suppose that the experimentally observed instability of the far field angular structure at monotonous change of the cavity aperture [2,3] is connected to this phenomenon as well.

\section{Conclusion}

As is seen, the analogy between a plane-plane cavity and a waveguide segment makes it possible to use a model of the closed cavity with partially transparent walls during the analysis of its properties, where the reflectance of side boundaries varies together with the inclination of plane waves (quasi-plane — at the small apertures). In this connection the plane waves can be considered as real components of intracavity field, the structure of which in this case is the subject of simple analytical calculations. As it was shown in the article, the simultaneous use of two cavity spatial characteristics — its optical $L_{\mathrm{o}}$ and effective $L_{e}$ lengths - is sufficient for taking into the objective account the role of cavity optical filling.

According to the initial task, the basic attention is given to determine the roles of parameters responsible for the angular structure of the output emission. It was established that the convergence angle of plane wave components of a mode is not only distinct from the diffraction one but also depends in some complex way on geometrical parameters of the cavity. It is necessary to pay attention to the fact that all ratios received in the article contain just really controllable values. This circumstance extremely simplifies an experimental verification of their reliability. The most perspective area of above ratio application is the aperture range characterized by the Fresnel number close to unity, when full round of the cavity by plane waves is realized for rather small number of their passes between mirrors.

\section{References}

1. Yu.A. Anan'ev, Optical cavity and laser beams // Moscow, Nauka, 1990, (in Russian).

2. S. Anokhov, Detailed study of plane-plane cavity fundamental mode far-field structure // Proc. SPIE, 4018, pp. 111-117, 1998.

3. S. Anokhov, On problem of the rigorous diffraction quantitative description // Sem. Phys. Quant. Elect. Optoel. 2(4), pp. 66-69 (1999).

4. A.G. Fox, T. Li, // Bell. System. Techn. J. 40, pp. 453-488 (1961).

5. L.A. Vainshtein, Open resonators and open waveguides // Moscow, Sovetskoe radio, 1976.

6. S. Anokhov, New approach to resonators with one-dimensional dispersion // Quant. Electr. 21, pp. 433-438 (1994). 